\title{
A model-predictive hypnosis control system under total intravenous anesthesia
}

\section{$\operatorname{AUTHOR}(\mathrm{S})$ :}

Sawaguchi, Yoshihito; Furutani, Eiko; Shirakami, Gotaro; Araki, Mituhiko; Fukuda, Kazuhiko

\section{CITATION:}

Sawaguchi, Yoshihito ... [et al]. A model-predictive hypnosis control system under total intravenous anesthesia. IEEE TRANSACTIONS ON BIOMEDICAL ENGINEERING 2008, 55(3): 874-887

\section{ISSUE DATE:}

2008-03

URL:

http://hdl.handle.net/2433/84539

\section{RIGHT:}

(c) 2008 IEEE. Personal use of this material is permitted. However, permission to reprint/republish this material for advertising or promotional purposes or for creating new collective works for resale or redistribution to servers or lists, or to reuse any copyrighted component of this work in other works must be obtained from the IEEE. 


\title{
A Model-Predictive Hypnosis Control System Under Total Intravenous Anesthesia
}

\author{
Yoshihito Sawaguchi*, Member, IEEE, Eiko Furutani, Gotaro Shirakami, Mituhiko Araki, Fellow, IEEE, and \\ Kazuhiko Fukuda
}

\begin{abstract}
In ambulatory surgery, anesthetic drugs must be administered at a suitable rate to prevent adverse reactions after discharge from the hospital. To realize more appropriate anesthesia, we have developed a hypnosis control system, which administers propofol as an anesthetic drug to regulate the bispectral index (BIS), an electroencephalography (EEG)-derived index reflecting the hypnosis of a patient. This system consists of three functions: 1) a feedback controller using a model-predictive control method, which can adequately accommodate the effects of time delays; 2) a parameter estimation function of individual differences; and 3) a risk control function for preventing undesirable states such as drug overinfusion or intraoperative arousal. With the approval of the ethics committee of our institute, 79 clinical trials took place since July 2002. The results show that our system can reduce the total amount of propofol infusion and maintain the BIS more accurately than anesthesiologist's manual adjustment.
\end{abstract}

Index Terms-Bispectral index (BIS), hypnosis control system, model-predictive control, propofol.

\section{INTRODUCTION}

D URING general anesthesia, anesthetic agents must be administered adequately to prevent intraoperative arousal and postoperative adverse reactions. This demand is crucial in ambulatory surgery because patients must remain in the hospital overnight if the adverse reactions are severe. To satisfy the demand, an intravenous anesthetic agent, propofol, is widely used for ambulatory anesthesia because propofol possesses favorable pharmacokinetic profiles [1] and shows reduced frequency of postoperative adverse reactions such as postoperative nausea and vomiting (PONV) [2]. The infusion rate of propofol during general anesthesia must be adjusted carefully to realize these merits. For that reason, numerous studies of automatic administration of propofol have been done [3]-[13]. Among them, target controlled infusion (TCI) system [3] is well known

Manuscript received August 11, 2006; revised August 15, 2007. The work of E. Furutani was supported in part by the Grant-in-Aid for Scientific Research (KAKENHI) from the Japanese Ministry of Education, Culture, Sports, Science and Technology under Grant 14750368 . This work was supported in part by the Center of Excellence for Research and Education of Fundamental Technologies in Electrical and Electronic Engineering, Kyoto University, Kyoto, Japan. Asterisk indicates corresponding author.

*Y. Sawaguchi is with the Department of Control Engineering, Kisarazu National College of Technology, 2-11-1 Kiyomidai-higashi, Kisarazu-shi, Chiba 292-0041, Japan (e-mail: sawaguti@ d.kisarazu.ac.jp).

E. Furutani is with the Department of Electrical Engineering, Kyoto University, Kyoto 615-8510, Japan.

G. Shirakami and K. Fukuda are with the Department of Anesthesia, Kyoto University Hospital, Kyoto 606-8507, Japan.

M. Araki is with the Matsue National College of Technology, Shimane 6908518, Japan.

Digital Object Identifier 10.1109/TBME.2008.915670 and now widely accepted. The TCI system administers propofol to regulate the propofol concentration in plasma or at the effect site of propofol close to a target level. It uses a pharmacokinetic model of propofol as a dynamic model to estimate and thereby regulate the concentration. Some researchers have advocated that the TCI system has sufficient performance for clinical use [4]. However, from the viewpoint of control engineering, the TCI system works as an open-loop controller and does not have tracking ability to a target. Consequently, anesthesiologists should adjust the target concentration suitably to cope with individual differences and noxious stimuli during surgery.

On the other hand, automatic infusion systems with feedback mechanisms have been studied [5]-[13] to handle the individual differences and the noxious stimuli adequately. They used an index derived from spontaneous electroencephalography, the bispectral index (BIS) [14], which is a satisfactory index of hypnosis under propofol anesthesia [15], [16]. In such systems, proportional-derivative (PD) or proportional-integral-derivative (PID) controllers [5]-[10], a model-based controller [11], [12], or a nonlinear adaptive controller [13] were used. However, most of those studies did not take into account the delays caused by the movement of propofol in an intravenous line, the distribution of propofol in blood vessels, and the signal processing time within the BIS monitor [17]. Struys et al. [12] took the delays into account in estimation of patient-specific parameters, but that system required much time, and the control strategy was unable to cope adequately with the delays. Bailey and Haddad [13] pointed out the need for consideration of the delays, because an undershoot of the BIS at the induction of anesthesia occurred in their nonlinear adaptive control system.

We have developed a hypnosis control system using the BIS as the index of hypnosis and propofol as the intravenous anesthetic agent. The system uses a model-predictive controller [18], which can rigorously take the delays into account. Moreover, it has a function of rapid estimation of individual pharmacodynamic parameters from the BIS response during the induction of anesthesia. With the approval of the Ethics Committee of the Kyoto University Graduate School and Faculty of Medicine, Kyoto, Japan, we applied the system to various kinds of ambulatory surgery at Kyoto University Hospital, Kyoto, Japan, and confirmed its accuracy in hypnosis control and effects of drug reduction. While a brief explanation of this system and main results were presented in [19] and [20], this paper describes the details of a model of BIS response to propofol infusion, the parameter estimation function, the control law, and the risk control function. Furthermore, the results of clinical trials are compared with those of other hypnosis control systems [5], [8]-[10], [12] and anesthesiologist's manual adjustment. 
TABLE I

PHARMACOKINETIC PARAMETER VALUES PUBLISHEd BY SCHÜTTLER AND IHMSEN [21]

\begin{tabular}{l|c||l|c}
\hline$V_{1}^{\text {con }}$ & $1.72 \mathrm{BW}^{0.71} \mathrm{age}^{-0.39} \mathrm{~L}$ & $V_{1}^{\text {bol }}$ & $4.49 \mathrm{BW}^{0.71}$ age $^{-0.39} \mathrm{~L}$ \\
\hline$V_{2}^{\text {con }}$ & $3.32 \mathrm{BW}^{0.61} \mathrm{~L}$ & $V_{2}^{\text {bol }}$ & $5.74 \mathrm{BW}^{0.61} \mathrm{~L}$ \\
\hline$V_{3}^{\text {con }}$ & $266 \mathrm{~L}$ & $V_{3}^{\text {bol }}$ & the same as $V_{3}^{\text {con }}$ \\
\hline$k_{1}^{\text {con }}$ & $0.0595 \mathrm{BW}^{0.75} \mathrm{~L} / \mathrm{min}($ age $\leq 60)$ & $k_{1}^{\text {bol }}$ & the same as $k_{1}^{\text {con }}$ \\
& $\left(0.0595 \mathrm{BW}^{0.75}-0.045 \mathrm{age}+2.7\right) \mathrm{L} / \mathrm{min}(\mathrm{age}>60)$ & & \\
\hline$k_{2}^{\text {con }}$ & $0.0969 \mathrm{BW}^{0.62} \mathrm{~L} / \mathrm{min}$ & $k_{2}^{\text {bol }}$ & $0.293 \mathrm{BW}^{0.62} \mathrm{~L} / \mathrm{min}$ \\
\hline$k_{3}^{\text {con }}$ & $0.0889 \mathrm{BW}^{0.55} \mathrm{~L} / \mathrm{min}$ & $k_{3}^{\text {bol }}$ & $0.0462 \mathrm{BW}^{0.55} \mathrm{~L} / \mathrm{min}$ \\
\hline
\end{tabular}

This paper is organized as follows. Section II is devoted to description of the model of the BIS response to propofol infusion. Individual differences in model parameters are also considered. In Section III, we explain a function for estimation of the individual differences, strategy and design of model-predictive controller, and a risk control function of the system. In Section IV, our clinical system and the results of the clinical trials are presented. Evaluation of the controller performance and discussion are made in Section V.

\section{Model of Hypnosis Change to Drug Infusion}

A mathematical model of the process is necessary to design an appropriate controller for a specific process. The accuracy of the model strongly affects the performance of control systems, especially when a model-based controller is used. In this section, we introduce a model of the BIS response to propofol infusion. The model is a series connection of three elements: a pharmacokinetic model, a time delay, and a pharmacodynamic model. In Sections II-A and II-B, detailed descriptions of respective elements are presented.

\section{A. Pharmacokinetic Model}

Pharmacokinetic models describe the dynamics of drug concentration in human body. We construct a pharmacokinetic model based on the population pharmacokinetic model given through a large-scale multicenter study by Schüttler and Ihmsen [21] because this model incorporates the patient's age and body weight (BW) and seems to be sufficiently reliable. However, the Schüttler-Ihmsen model does not include the effect site [22] that relates directly to the BIS. Furthermore, the model parameters for continuous infusion of the Schüttler-Ihmsen model differ from those for a bolus. Considering these factors, we propose a unified model that can accommodate both bolus and continuous infusion and attach the effect site compartment to the unified model.

The original version of the Schüttler-Ihmsen model is given as (1), shown at the bottom of the page. Here, $x_{i}$ is the concentration of propofol in compartment $i$; compartments 1,2 , and
3 correspond, respectively, to the central, shallow peripheral, and deep peripheral compartment. In addition, $u$ is the infusion rate of propofol, and $k_{i}$ and $V_{i}$ are the clearance and volume of compartment $i$, respectively, given as functions of the patient's age and BW, as in Table I. The superscripts bol and con of the parameters in Table I, respectively, designate administration by bolus and continuous infusion.

Based on (1), we constructed a unified model under the following assumptions.

- The parameter values of the unified model to continuous infusion, except for those of the effect site, are given by the parameter values for continuous infusion in Table I, because anesthesia is usually maintained by continuous infusion.

- In the bolus case, infused propofol moves directly to the shallow peripheral compartment and to the central compartment, as shown in Fig. 1.

Physiologically, a bolus of propofol reduces hepatic blood flow [23], which decreases the clearance of the central compartment. Consequently, the bolused propofol remains in the patient's body for a longer time than in continuous-infusion cases. Although this phenomenon might be considered by a nonlinear clearance, such nonlinearity increases the model complexity. Furthermore, the exact form of the nonlinearity has never been shown. For those reasons, we introduce a linear model that imitates this phenomenon by assuming that a fraction of the bolused propofol accumulates in the shallow peripheral compartment, and fades after the transition to the central compartment.

- The volume $V_{4}$ of the effect site compartment is one hundredth of the central compartment. Namely, $V_{4}$ is set to $V_{4}=V_{1}^{\text {con }} / 100$.

Many studies have subsumed that this volume is negligible. However, that consideration relies on the assumption that a drug is metabolized or eliminated at the effect site. That assumption seems to be irrelevant for hypnotic agents. Furthermore, the assumption changes the relative degree of a

$$
\frac{d}{d t}\left[\begin{array}{l}
x_{1}(t) \\
x_{2}(t) \\
x_{3}(t)
\end{array}\right]=\left[\begin{array}{ccc}
-\frac{k_{1}+k_{2}+k_{3}}{V_{1}} & \frac{k_{2}}{V_{1}} & \frac{k_{3}}{V_{1}} \\
\frac{k_{2}}{V_{2}} & -\frac{k_{2}}{V_{2}} & 0 \\
\frac{k_{3}}{V_{3}} & 0 & -\frac{k_{3}}{V_{3}}
\end{array}\right]\left[\begin{array}{l}
x_{1}(t) \\
x_{2}(t) \\
x_{3}(t)
\end{array}\right]+\left[\begin{array}{c}
\frac{1}{V_{1}} \\
0 \\
0
\end{array}\right] u(t)
$$




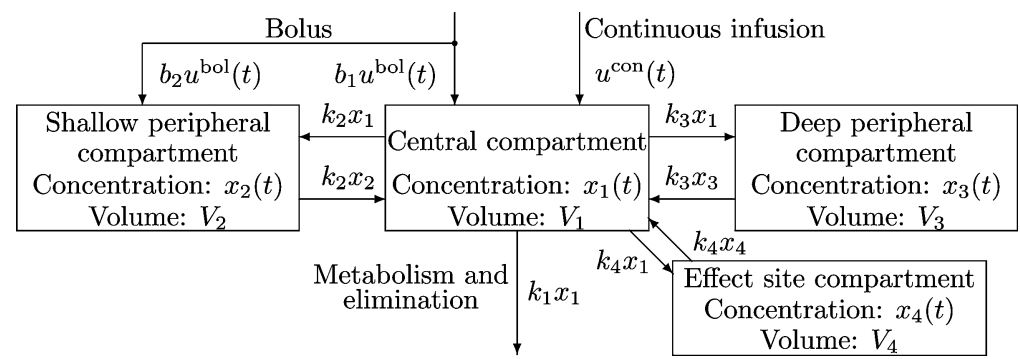

Fig. 1. Three-compartment pharmacokinetic model with the effect site and a direct route to a shallow peripheral compartment for a bolus.

transfer function of the process. For those reasons, we did not neglect the volume of the effect site compartment, and regarded it to one hundredth of the central compartment.

The constructed pharmacokinetic model is given as (2), shown at the bottom of the page, with $x(t)=$ $\left[\begin{array}{llll}x_{1}(t) & x_{2}(t) & x_{3}(t) & x_{4}(t)\end{array}\right]^{T}$, where $x_{4}$ is the propofol concentration in the effect site compartment, $u^{\mathrm{bol}}(t)$ is the bolus rate, and $u^{\operatorname{con}}(t)$ is the continuous infusion rate. Constants $b_{1}$ and $b_{2}$ are input coefficients for the bolus, and they are given by solving the minimization problem of the quadratic error between $x_{1}(t)$ after a single bolus (an impulse input) of the Schüttler-Ihmsen model and that of (2). The clearance $k_{4}$ of the effect site compartment was determined from preliminary data collected from 47 patients [male/female 16/31, age $48 \pm$ 18 years (mean $\pm \mathrm{SD}$ ), BW $57 \pm 11 \mathrm{~kg}]$ under various kinds of ambulatory surgery. In these measurements, propofol was administered at the rate of $120 \mathrm{mg} / \mathrm{kg} / \mathrm{h}$ for the first $1 \mathrm{~min}$, then at the rate of $10 \mathrm{mg} / \mathrm{kg} / \mathrm{h}$ for the subsequent 2 or $3 \mathrm{~min}$. Thereafter, the rate was adjusted to maintain the BIS within the range of 40-60 until surgery was completed. The values of the BIS and infusion rate were obtained in every $1 \mathrm{~s}$. Using these measurements, $k_{4}$ was set to $0.12 \mathrm{~L} / \mathrm{min}$ to make the median peak time [24] of the effect site concentration calculated using (2) coincide with the time between the beginning and the peak of the averaged BIS decrease among the patients.

In the following, we treat the infusion whose rate is greater than $u^{\text {th }}$, the threshold rate of the continuous infusion, as a bolus. That is, we define $u^{\mathrm{con}}(t)$ and $u^{\mathrm{bol}}(t)$ as

$$
\begin{aligned}
& u^{\mathrm{con}}(t)= \begin{cases}u(t), & \text { for } u(t) \leq u^{\text {th }} \\
0, & \text { otherwise }\end{cases} \\
& u^{\mathrm{bol}}(t)= \begin{cases}0, & \text { for } u(t) \leq u^{\text {th }} \\
u(t), & \text { otherwise. }\end{cases}
\end{aligned}
$$

The threshold rate $u^{\text {th }}$ is set to $20 \mathrm{mg} / \mathrm{kg} / \mathrm{h}$, which is twice the upper bound of the adequate infusion rate recommended by the supplier (AstraZeneca, Osaka, Japan), because we consider that, in most situations, the rate $u^{\text {th }}$ is sufficiently large for maintenance of anesthesia. In all the collected data, the continuous infusion rate of propofol was less than $u^{\mathrm{th}}$.

Fig. 2 shows responses of $x_{1}(t)$ to a bolus and continuous infusion for the Schüttler-Ihmsen model and the constructed unified model for a 40 -year-old patient whose BW is $60 \mathrm{~kg}$. These figures show that the unified model provides adequate responses both to the bolus and continuous infusion over the whole time domain. Similar results were obtained for patients of all ages and BWs.

\section{B. Time Delays and the Pharmacodynamic Model}

In this section, we present a detailed description of delays and a pharmacodynamic model, and estimate their parameter values from preliminary measurements. Furthermore, the necessity for consideration of individual differences is described.

The response of the BIS to propofol infusion includes considerable time delays, which are caused by movement of propofol from a three-way stopcock to the patient's body in an intravenous fluid line, distribution of propofol in blood vessels (the central compartment), and calculation time of the BIS in the BIS monitor (approximately 15-60 s [17]).

The process is a single-input-single-output system. Therefore, we consider a single-output delay $L_{c}$ whose length is the sum of the delays described previously. That is, the current BIS value $\operatorname{BIS}(t)$ is determined by the past value $y(t)$ of the effect site concentration

$$
y(t)=C x\left(t-L_{c}\right)
$$

where $C=\left[\begin{array}{llll}0 & 0 & 0 & 1\end{array}\right]$.

A pharmacodynamic model describes the relationship between the propofol concentration in the effect site compartment

$$
\frac{d x(t)}{d t}=\left[\begin{array}{c}
-\frac{k_{1}^{\mathrm{con}}+k_{2}^{\mathrm{con}}+k_{3}^{\mathrm{con}}+k_{4}}{V_{1}^{\mathrm{con}}} \\
\frac{k_{2}^{\mathrm{con}}}{V_{2}^{\mathrm{con}}} \\
\frac{k_{3}^{\mathrm{con}}}{V_{3}^{\mathrm{con}}} \\
\frac{k_{4}}{V_{4}}
\end{array}\right.
$$

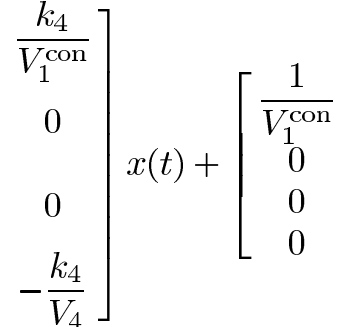

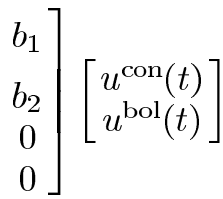




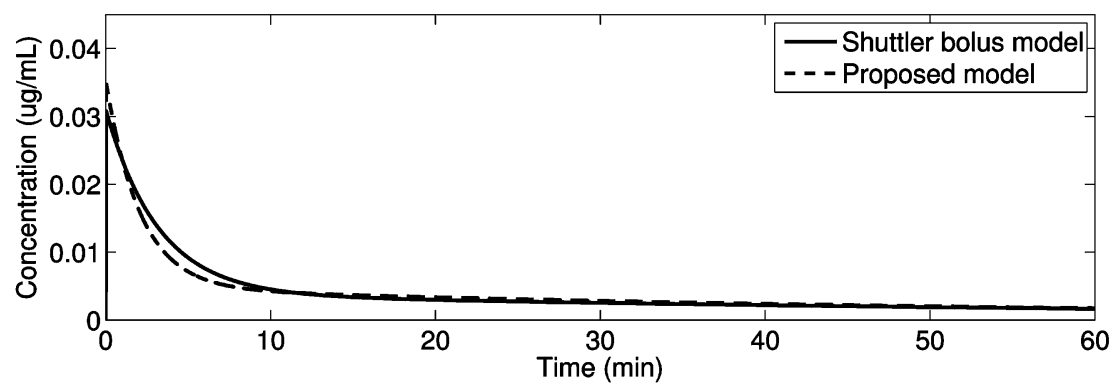

(a)

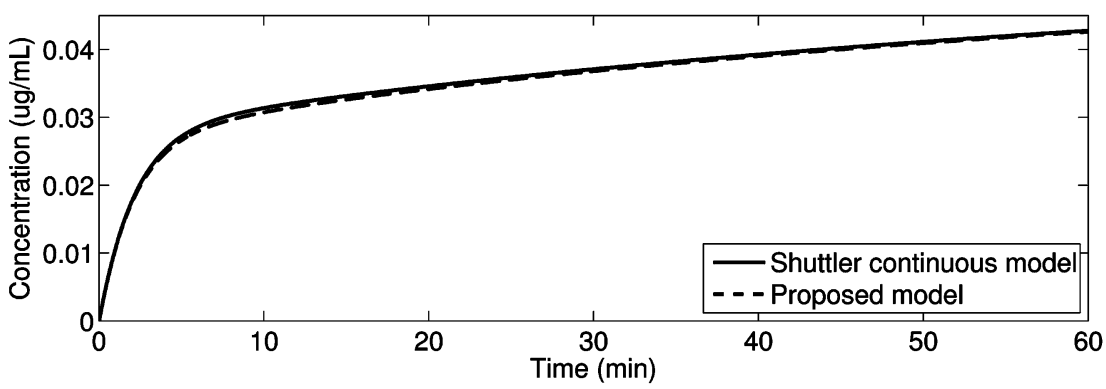

(b)

Fig. 2. Plasma propofol concentration of the conventional pharmacokinetic model published by Schüttler and Ihmsen [21] and the proposed model. (a) Plasma propfol concentration to $1-\mathrm{mg} / \mathrm{kg}$ bolus. (b) Plasma propofol concentration to $10-\mathrm{mg} / \mathrm{kg} / \mathrm{h}$ continuous infusion.

and the BIS. We use the sigmoidal $E_{\max }$ model [25] because it is widely accepted in pharmacodynamic studies of propofol [26], [27]. This model is given as

$$
\operatorname{BIS}^{\text {model }}(t)=E_{0}-E_{\max } \frac{y^{\gamma}(t)}{y^{\gamma}(t)+c_{50}^{\gamma}}
$$

where $\operatorname{BIS}^{\text {model }}(t)$ is the current BIS, $E_{0}$ is the BIS on awakening, $E_{\max }$ is the maximum-effect intensity, $c_{50}$ is the propofol concentration corresponding to the $E_{\max } / 2$, and $\gamma$ is the Hill coefficient.

Next, we estimate the actual values of the delay length and pharmacodynamic parameters from the 47 data sets described in Section II-A. These estimates are used for validation of a closed-loop controller.

To begin with, the delay length is estimated for each measurement. First, propofol concentration in the effect-site compartment is calculated from the propofol infusion rate $u(t)$ and (2). Then, the estimate of the delay length $\hat{L}_{c}$ is determined through trial and error so that the relation between $y(t)$ and $\operatorname{BIS}(t)$ during the induction (the first $120 \mathrm{~s}$ after the start of infusion) approaches the relation during the awakening period (time period after the stop of infusion), as presented in Fig. 3(a) and (b).

Under the assumption that $E_{\max }=E_{0}$, which means the BIS tends to 0 when propofol concentration in the effect site becomes infinite, other parameters are estimated as follows. $E_{0}$ is set to the maximum BIS value before the start of infusion. The constants $c_{50}$ and $\gamma$ are determined through exhaustive search for the minimum of a model fitness measure

$$
J=\sum_{i=1}^{3} \sum_{t \in T_{i}} \frac{\left[\mathrm{BIS}^{\text {model }}\left(t+\hat{L}_{c}\right)-\operatorname{BIS}\left(t+\hat{L}_{c}\right)\right]^{2}}{\text { length }\left(T_{i}\right)}
$$

where $T_{1}, T_{2}$, and $T_{3}$ mean the induction, awakening, and maintenance period that are defined, respectively, as the period up to $120 \mathrm{~s}$ from the start of infusion, the period after the end of infusion, and the period between induction and awakening periods. Moreover, length $\left(T_{i}\right)$ represents the number of data points in $T_{i}$.

In the preliminary 47 data sets, the estimates of $L_{c}, E_{0}, c_{50}$, and $\gamma$ are $68 \pm 34 \mathrm{~s}, 97.3 \pm 0.8,3.90 \pm 1.05 \mu \mathrm{g} / \mathrm{mL}$ and $1.81 \pm$ 0.67 , respectively. The mean and standard deviation of the measure $J$ for each measurement are 95.7 and 52.9, respectively.

The measure with fixed parameter values is calculated to consider the effect of individual differences. Substituting the mean values of the estimates into $L_{c}, E_{0}, c_{50}$, and $\gamma$, the mean and standard deviation of measure $J$ are 266.2 and 151.9 , respectively. The mean is significantly larger than that with the individualized parameters. Therefore, we should consider the individual differences of these parameters.

Additionally, we introduce a linear pharmacodynamic model as

$$
\operatorname{BIS}^{\operatorname{lin}}(t)=k^{\operatorname{lin}} y(t)+\mathrm{BIS}^{0}
$$

and compare the model fitness for the nonlinear and linear model. The parameters $k^{\text {lin }}$ and BIS $^{0}$ are identified individually using least-squares method under the same measure of model fitness. The identified values of $k^{\text {lin }}$ and $\mathrm{BIS}^{0}$ are, respectively, $-11.5 \pm 3.9$ and $94.7 \pm 11.7$. The mean and standard deviation of the measure $J$ are 112.6 and 57.3, respectively. The mean is larger than that with the nonlinear model with individualized parameters. Therefore, the nonlinear pharmacodynamic model (8) is more adequate than the linear one. In the following, we consider the nonlinear pharmacodynamic model using individualized parameters. 


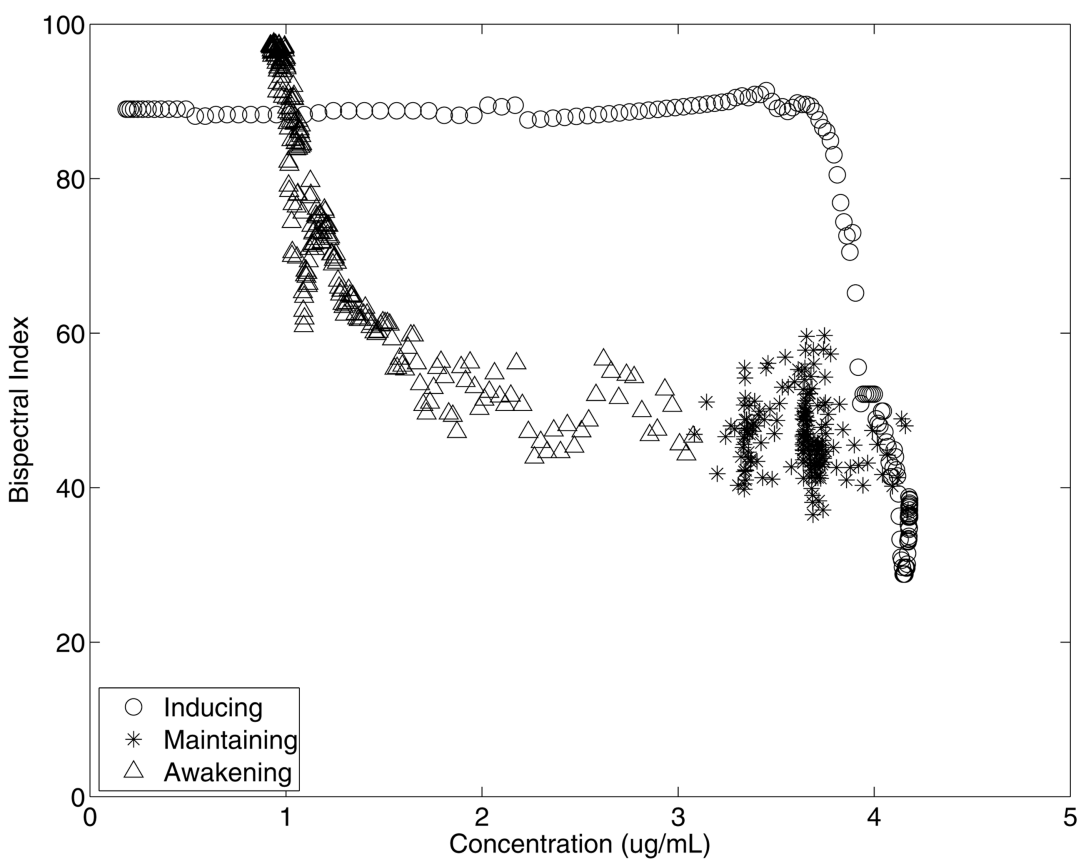

(a)

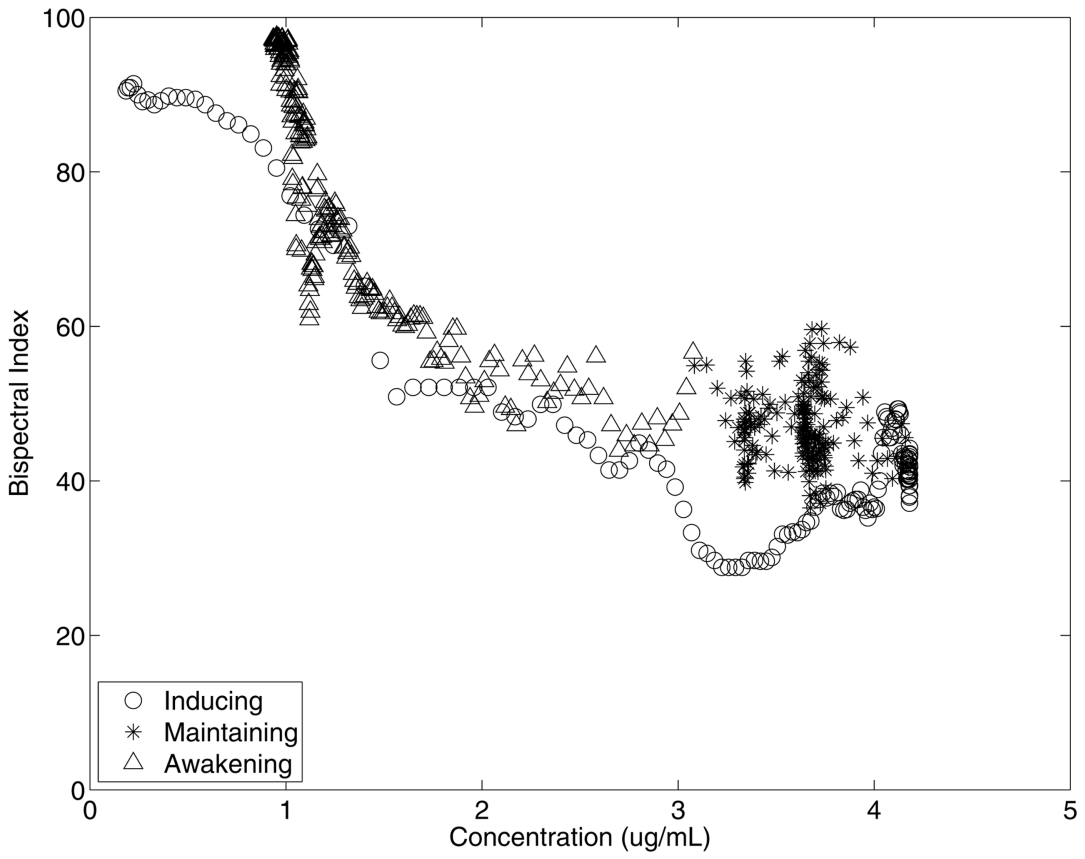

(b)

Fig. 3. BIS values versus effect site concentrations. (a) When the delay is not considered $\left(\hat{L}_{c}=0\right)$, the relation between BIS and the effect site concentration shows a hysteresis loop. (b) When the delay is considered adequately $\left(\hat{L}_{c}=60 \mathrm{~s}\right)$, the relation between BIS and the effect site concentration during the induction period approaches the relation during the awakening period.

\section{HYPNOSIS CONTROL SYSTEM}

In this section, our hypnosis control system is explained in detail. The main components of the control system are an estimation function of individual parameters during induction of anesthesia, a model-predictive controller, and a risk control function for prevention of undesired states. In Sections III-A-III-C, the detailed descriptions of these components are given.

\section{A. Parameter Estimation During Induction}

As presented in Section II-B, the individual differences in the pharmacodynamic parameters and the delay length are quite large. The differences might degrade the hypnosis control performance if they are not treated adequately. Therefore, we developed a parameter estimation function that estimates the pharmacodynamic parameters and the delay length from the 
TABLE II

Permissible RANGES AND DEFaUlt VALUES For PARAMETER Estimation

\begin{tabular}{l||c|c|c|c|c}
\hline & $E_{0}$ & $E_{\max }$ & $\gamma$ & $c_{50}(\mu \mathrm{g} / \mathrm{mL})$ & $L_{c}(\mathrm{~s})$ \\
\hline \hline Upper bound & 98.0 & 98.0 & 4.20 & 7.70 & 170 \\
\hline Lower bound & 87.0 & 87.0 & 0.70 & 1.70 & 30 \\
\hline Default value & 97.0 & 97.0 & 1.87 & 3.75 & 73 \\
\hline
\end{tabular}

response of the BIS to propofol infusion during induction of anesthesia.

For induction of propofol anesthesia, propofol is usually administered as sequential bolus and continuous infusion [28] to anesthetize patients quickly. According to the widely accepted procedure for propofol-induced anesthesia, our hypnosis control system administers propofol at the respective rates of $120 \mathrm{mg} / \mathrm{kg} / \mathrm{h}$ and $10 \mathrm{mg} / \mathrm{kg} / \mathrm{h}$ for the first $1 \mathrm{~min}$ and the subsequent 2 or $3 \mathrm{~min}$. In the end of this induction period, the individual delay length $L_{c}$ and pharmacodynamic parameters $E_{0}, E_{\max }, c_{50}$, and $\gamma$ are estimated from the response of the BIS and the calculated effect site concentration. Immediately after the parameter estimation is completed, parameters of the controller are adjusted using the estimation result, and feedback control starts.

In the following, we explain important details of the parameter estimation method. First, $E_{0}$ and $E_{\max }$ are determined as the maximum value of the BIS before the bolus of propofol. Next, the delay length $L_{c}$ is determined using an estimation equation

$$
L_{c}=0.932 T_{30}-41.5 \mathrm{~s}
$$

where $T_{30}$ is the time when $\operatorname{BIS}(t)$ reaches $\left(E_{0}-30\right)$. Equation (11) is derived from least-squares fitting of $T_{30}$ and the delay $\hat{L}_{c}$, estimated using trial and error, in the preliminary 47 data sets, because they are strongly correlated: the correlation coefficient is 0.956 . The standard deviation of the estimation error $\left(L_{c}-\right.$ $\left.\hat{L}_{c}\right)$ is $13.8 \mathrm{~s}$. Then, $c_{50}$ and $\gamma$ are identified using least-squares fitting based on

$$
\log \left(\frac{E_{0}-\operatorname{BIS}(t)}{\operatorname{BIS}(t)+E_{\max }-E_{0}}\right)=\gamma \log y(t)-\gamma \log c_{50}
$$

using measurements obtained during the BIS descending period from the start of infusion up to $\left(L_{c}+120\right)$ s. The above equation is derived from (8), as in the Appendix. Using this method, the delay length and pharmacodynamic parameters of each are estimated during the induction period.

This parameter estimation works well for almost all cases. However, the estimator must respond to device failures or severely abnormal measurements. If any obtained parameter value is out of the corresponding range shown in Table II, the parameter is set at the upper or lower bound of the range. If the measured data are unreliable, in other words, if the signal quality of electroencephalogram is fatally low or the BIS is higher than $\left(E_{0}-30\right)$ during the $180 \mathrm{~s}$ after the start of the bolus, the parameters are set to the default values shown in Table II. The upper and lower bounds and default values are determined to guarantee the robust stability of control system under possible parameter differences estimated from the preliminary measurements.

\section{B. Model-Predictive Controller}

In this section, we introduce a model-predictive controller [18] for controlling the hypnosis level of patients during general anesthesia. As described in Section II-B, the model of the BIS response to propofol infusion includes a delay. Furthermore, the infusion rate must be nonnegative. We designed a model-predictive controller to handle the delay and input constraint appropriately. In the following, we explain the salient details of the model-predictive controller that we use in our hypnosis control system.

1) Linearization of Model: The model of the BIS response to propofol infusion includes the nonlinear pharmacodynamic model. The model is linearized by an inverse of the pharmacodynamic model to simplify computation in the model-predictive controller.

We consider the controlled process as the linearized system obtained by adding the inverse of the pharmacodynamic model to the output side of the process. That is, we regard the effect site concentration estimated from the measured BIS as the controlled variable

$$
y^{\text {measured }}(t)=c_{50}\left[\frac{E_{0}-\operatorname{BIS}(t)}{E_{\max }-E_{0}+\operatorname{BIS}(t)}\right]^{1 / \gamma} .
$$

In a similar way, the target value for the model-predictive controller is set to

$$
y^{\text {target }}=c_{50}\left[\frac{E_{0}-\text { BIS }^{\text {target }}}{E_{\max }-E_{0}+\text { BIS }^{\text {target }}}\right]^{1 / \gamma}
$$

where BIS ${ }^{\text {target }}$ is the target value of the BIS. Although the nominal values of parameters used in (13) and (14) might differ from the actual value of the process, the BIS coincides with its target value when the controlled variable $y^{\text {measured }}(t)$ reaches its target value $y^{\text {target }}$ because the pharmacodynamic model and its inverse are one-to-one mappings.

2) Control Law: The control law of our model-predictive controller is shown here. First, we introduce notation and assumptions. We, respectively, describe the starting time of propofol infusion and the present time as $t=0$ and $t=k T$, where $T$ is the sampling period of the controller. We assume that the initial state is zero, $x(\tau)=0$ for $\tau \leq 0$, because no propofol exists in the patient's body before the start of propofol infusion. Furthermore, we assume that the bolus of propofol will not be administered after the present time and that the infusion rate of propofol will be changed only at every sampling instant. That is, the future inputs are assumed to be

$$
\begin{aligned}
u(t) & =u_{l}, \quad l T \leq t<(l+1) T, \quad l=k, k+1, \ldots \\
0 & \leq u_{l} \leq u^{\text {th }}, \quad l=k, k+1, \ldots
\end{aligned}
$$

where $u_{l}$ for $l=k, k+1, \ldots$ is the infusion rate of propofol during the future period $[l T,(l+1) T)$.

Under these assumptions, the future output is predicted by the model. From the initial condition $x(0)=0$ and the past input $u(t)$ for $t \in[0, k T)$, the future output $y^{\operatorname{model}}(k T+\tau)$ of the nominal model for $\tau \geq 0$ is predicted as a function of future inputs $u_{k}, u_{k+1}, \ldots$, using (2) and (7). Additionally, a constant disturbance

$$
e_{k}=y^{\text {measured }}(k T)-y^{\text {model }}(k T)
$$


is introduced to suppress the output disturbance and the error resulting from model mismatches [18]. Consequently, the predicted output is given as

$$
\hat{y}(k T+\tau)=y^{\text {model }}(k T+\tau)+e_{k}, \quad \tau \geq 0 .
$$

Next, we introduce the reference trajectory that the predicted output should track as closely as possible. We use the reference trajectory for the effect-site concentration to avoid aggressive control action. Using the target value $y^{\text {target }}$ and the predicted output $\hat{y}\left(k T+L_{c}\right)$ at time point $k T+L_{c}$, the reference trajectory $y^{\text {ref }}(k T+\tau)$ for $\tau \geq L_{c}$ is set as

$$
\begin{aligned}
y^{\mathrm{ref}}(k T+\tau)=\left(1-e^{\left(\tau-L_{c}\right) / T^{\mathrm{ref}}}\right) y^{\text {target }} & \\
& +e^{\left(\tau-L_{c}\right) / T^{\mathrm{ref}}} \hat{y}\left(k T+L_{c}\right)
\end{aligned}
$$

where $T^{\mathrm{ref}}$ is a time constant of the reference trajectory.

Then, future input values $u_{k}, u_{k+1}, \ldots$ are calculated as the solution of the following optimization problem:

$$
\begin{array}{r}
\min _{u_{k}, \ldots, u_{k+N_{M}-1}} J=\sum_{l=k+1}^{k+N_{P}}\left[y^{\mathrm{ref}}\left(l T+L_{c}\right)-\hat{y}\left(l T+L_{c}\right)\right]^{2} \\
+\lambda \sum_{l=k}^{k+N_{M}-1} u_{l}^{2}
\end{array}
$$

subject to

$$
\begin{aligned}
0 & \leq u_{l} \leq u^{\text {th }}, \quad l=k, k+1, \ldots, k+N_{M}-1 \\
u_{l} & =u_{k+N_{M}-1}, \quad l=k+N_{M}, k+N_{M}+1, \ldots
\end{aligned}
$$

where $N_{M}$ is the length of a control horizon, $N_{P}$ is the length of a prediction horizon, and $\lambda$ is a weighting coefficient for the input to the output error.

Finally, the controller applies the first element $u_{k}$ of the future inputs, which were obtained by solving the optimization problem (20), to the controlled process, and repeats this procedure at each sampling time.

3) Controller Design: In this section, we give specifications of the control system and design the model-predictive controller. The hypnosis level of the patient should approach quickly to the target value and then be maintained around the target. Furthermore, the control system must be robustly stable for individual differences among patients, although the effect of the differences might be suppressed partly by the parameter estimation function. Taking these requirements into account, the specifications of the hypnosis control system are given for patients whose age, $\mathrm{BW}$, and delay length are in the ranges of $18-80$ years, $40-100 \mathrm{~kg}$, and $30-150 \mathrm{~s}$, respectively. First, the 5\% settling time of the model-predictive control system should be less than $15 \mathrm{~min}$ to provide clinically acceptable responses. Second, the control system should be stable for $\bar{L}_{c} \in\left[0.1 L_{c}, 1.9 L_{c}\right]$ and $K \in[0,2.1]$, where $\bar{L}_{c}$ and $L_{c}$ are actual and nominal delay lengths and $K$ is the relative gain between the actual and nominal processes. These ranges are given by the three sigma limits of relative mismatch of delay estimate in Section III-A and those of linear gain estimated in Section II-B, respectively.
To satisfy those specifications for every patient model in the targeted characteristic group, the controller parameters $T, N_{P}$, $N_{M}, T^{\text {ref }}$, and $\lambda$ are configured. The sampling period $T$ of the controller is set to $10 \mathrm{~s}$ under a mechanical restriction of a syringe pump. The length of the control horizon $N_{M}$ is set to one because the control system must have sufficient robustness. The time constant $T^{\text {ref }}$ of the reference trajectory is set to $245 \mathrm{~s}$ so that the settling time of the reference trajectory meets the specification of settling time. The length of the prediction horizon $N_{P}$ and the weighting coefficient $\lambda$ are set to $N_{P}=30$ and $\lambda=10^{-4}(\mu \mathrm{g} / \mathrm{mL})^{2} /(\mathrm{mg} / \mathrm{kg} / \mathrm{h})^{2}$ by trial and error to provide a sufficient robust stability margin of the closed-loop system.

Typical nominal responses are shown in Fig. 4, which shows the nominal step response of the closed-loop system and the nominal closed-loop response after the sequential bolus and continuous infusion for a 40 -year-old, $60-\mathrm{kg}$ patient with mean parameter values described in Section II-B. We can confirm that adequate control is achieved and that the settling time is less than $15 \mathrm{~min}$. A typical result of robust stability analysis for the same patient model is shown in Fig. 5. This figure is obtained from the Nyquist stability condition with the assumption that the modeling errors exist only in the linear gain and the delay length [29]. We can confirm that the robust stability region is sufficiently large to satisfy the specified requirement.

We repeated simulations and analysis for models of patients without or with model mismatches on pharmacokinetic and pharmacodynamic parameters and delay length. The mismatches were given as normally distributed random values, whose mean and standard derivation were the nominal values and one fifth of the nominal values for pharmacokinetic parameters, and the mean and standard derivation of the estimates in the preliminary 47 data sets for pharmacodynamic parameters and delay length, respectively. Under these model mismatches, we confirmed that our hypnosis control system satisfies the given specifications.

\section{Risk Control Function}

In the usual mode, the infusion rate of propofol is adjusted by the model-predictive controller to maintain the BIS around the target value. However, unmodeled surgical disturbances and sensitive autonomic nervous system might cause undesirable states, such as intraoperative arousal, hypotension, and bradycardia. To prevent these undesirable states as well as overinfusion of propofol, we implement a rule-based risk control function that imitates countermeasures taken by anesthesiologists for such states because the model-predictive controller cannot respond quickly to such unmodeled factors. In the following, we explain each risk-avoiding action.

1) Prevention of Propofol Overinfusion: To avoid adverse reactions of propofol overinfusion, such as a delay of arousal or PONV, the upper bound of the infusion rate is set to $20 \mathrm{mg} / \mathrm{kg} / \mathrm{h}$, except the bolus for a countermeasure to intraoperative arousal. Moreover, the bolus dose for the countermeasure to intraoperative arousal is limited to less than $U_{\max }=1 \mathrm{mg} / \mathrm{kg}$, as explained in the following.

2) Countermeasure to Intraoperative Arousal: The system administers a bolus of propofol to prevent intraoperative arousal when the BIS rises abnormally. The dose and rate of the bolus 

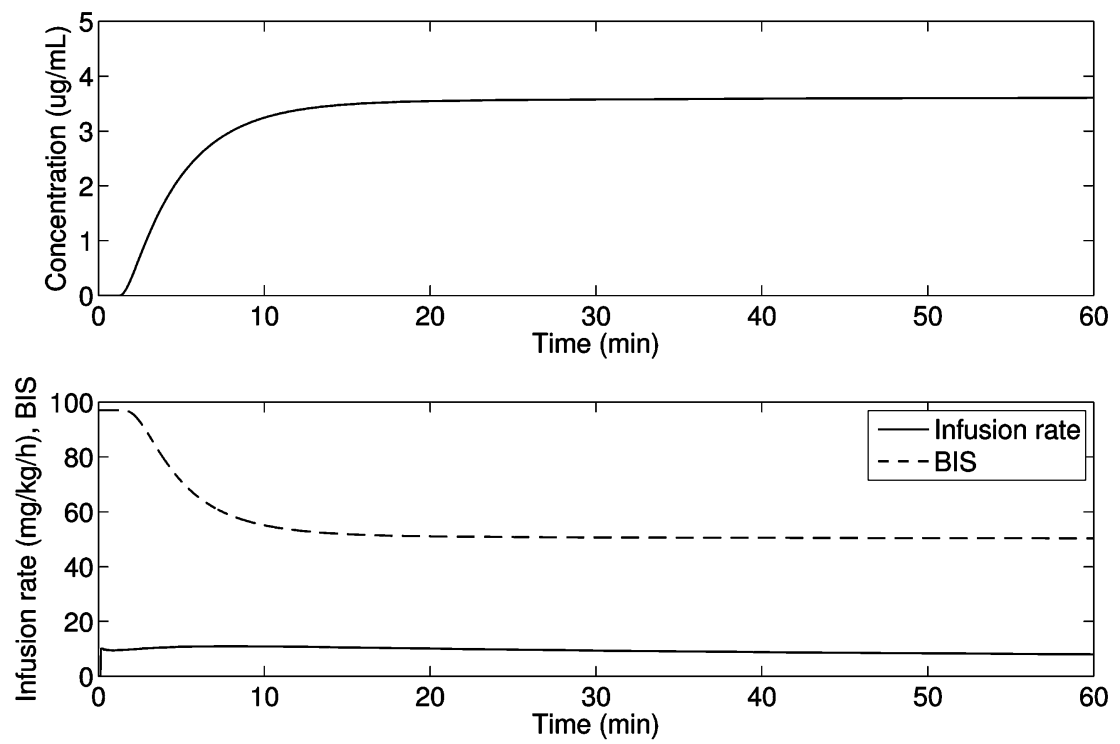

(a)
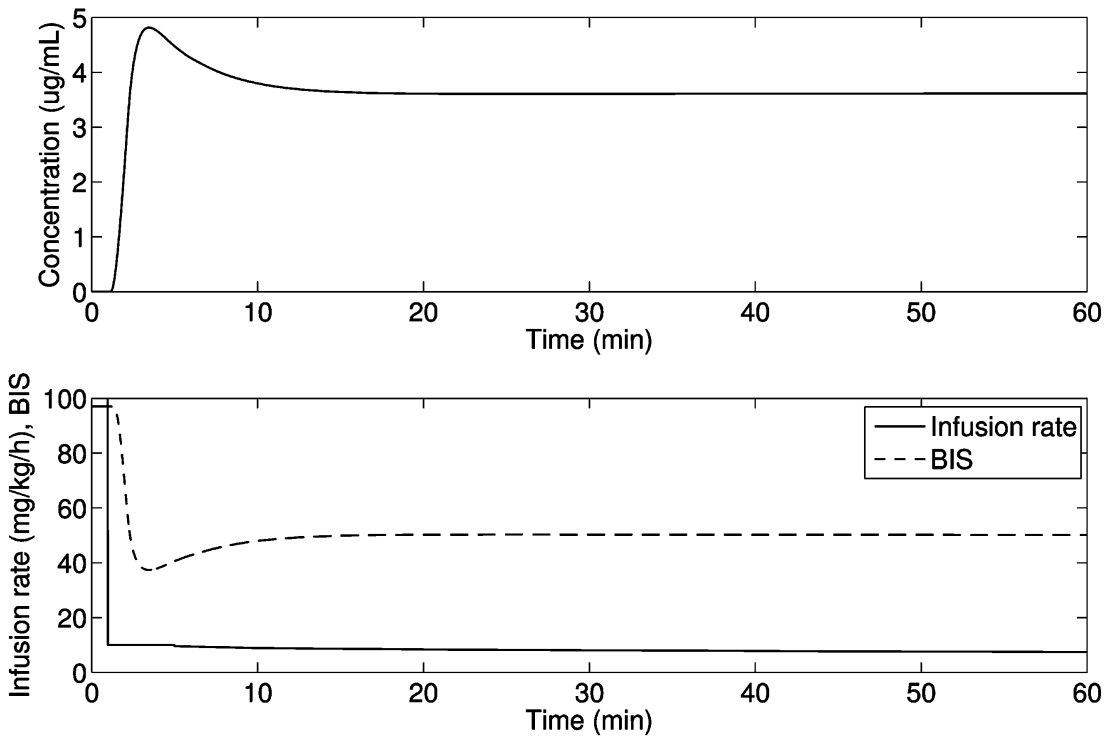

(b)

Fig. 4. Simulation results of the closed-loop system with a model of patient whose age, BW, and delay length are 40 years, $60 \mathrm{~kg}$, and $70 \mathrm{~s}$. In each figure, the upper panel shows propofol concentrations at the effect site, and the lower panel shows the infusion rate of the propofol (solid line), and the BIS (dashed line). The setpoints of the concentration and the BIS are set, respectively, to $3.6 \mu \mathrm{g} / \mathrm{mL}$ and 50. (a) Nominal step response. (b) Nominal response after the sequential bolus and continuous infusion.

are determined based on the BIS rise $\Delta \mathrm{BIS}(t)=(\mathrm{BIS}(t)-$ BIS $\left.^{\text {target }}\right)$, by the following procedure.

- When the bolus at the induction is completed, $U_{\text {sum }}$ is set to $2 \mathrm{mg} / \mathrm{kg}$. Here, $U_{\text {sum }}$ is a fictitious dosage, which corresponds to the effect of past boluses.

- During continuous infusion, $U_{\text {sum }}$ is decreased exponentially with a time constant of $195 \mathrm{~s}$.

- When $\Delta \mathrm{BIS}(t)$ is greater than 15 , the system sets a limit dose $U_{\mathrm{bol}}$ of the following bolus to $\left(U_{\max }-U_{\text {sum }}\right)$, and starts a bolus at the rate of $30 \mathrm{mg} / \mathrm{kg} / \mathrm{h}$.

- During the bolus, the bolus rate is increased to 60,90 , and $120 \mathrm{mg} / \mathrm{kg} / \mathrm{h}$ if the respective $\Delta \mathrm{BIS}(t)$ are greater than 25 , 35 , and 45 . On the other hand, the rate is decreased to 90 , 60 , and $30 \mathrm{mg} / \mathrm{kg} / \mathrm{h}$ if $\Delta \mathrm{BIS}(t)$ falls under 40,30 , and 20 , respectively. If $\Delta \mathrm{BIS}(t)$ falls below 10 or the bolus dose reaches $U_{\mathrm{bol}}$, the bolus is terminated, and the bolus dose is added to $U_{\text {sum }}$.

3) Management for Hypotension and Bradycardia: To cope with adverse reactions of propofol to the patient's cardiovascular system, the system continuously monitors the noninvasive systolic pressure and pulse rate. When these values respectively fall below $70 \mathrm{mmHg}$ and $40 \mathrm{~b} / \mathrm{min}$, the system sounds an alarm and decreases the propofol infusion rate according to a predicted awakening time. The predicted awakening time is calculated from (2) and (7) as the time for $y(t)$ to reach $y_{80}$, which is the effect site propofol concentration corresponding to BIS $=80$, under the assumption that $u(t+\tau)=0(\tau \geq 0)$. The propofol infusion rate is set to zero if the predicted awakening time is 


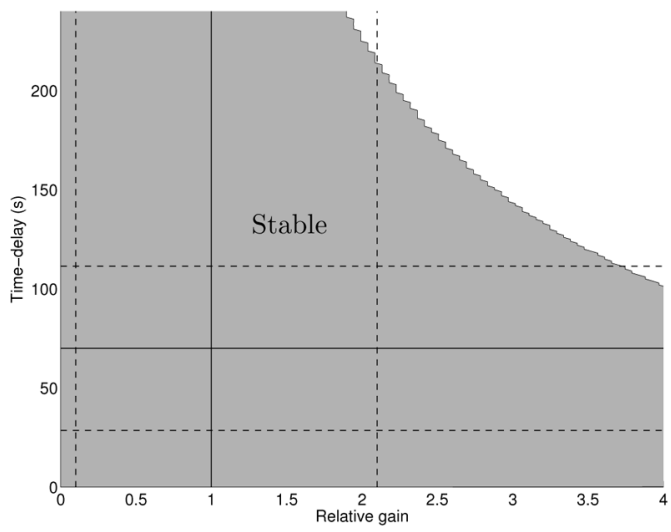

Fig. 5. Stability region of the closed-loop system in the gain-time-delay plane for a model of patient whose respective age, BW, and delay length are 40 years, $60 \mathrm{~kg}$, and $70 \mathrm{~s}$. Solid lines mean the nominal values and dashed lines mean the three sigma limits.

longer than $4 \mathrm{~min}$. Otherwise, the propofol infusion rate is set to a half of the calculated rate by the model-predictive controller.

4) Countermeasure to BIS Noise: The BIS is calculated from the measured EEG. When an electric knife is used, a signal quality of the EEG worsens because of electrical noise; sometimes the BIS output is suspended. Moreover, when the myogenic potential appears frequently on the EEG, the BIS output is also suspended. During the suspension, the model-predictive controller cannot obtain the measured output. Therefore, the controller determines the propofol infusion rate assuming that the step disturbance $e_{k}$ in (18) is the same as that at one step before, namely, $e_{k}=e_{k-1}$.

\section{Clinical Trials}

\section{A. Implementation}

Implementation of the hypnosis control system is shown in Fig. 6. This system measures the BIS, the noninvasive systolic blood pressure, and the pulse rate. The BIS is measured using a BIS monitor (A-2000; Aspect Medical Systems, Newton, MA). The noninvasive systolic blood pressure and the pulse rate are measured using a central monitoring system (Agilent Technologies, Palo Alto, CA). The propofol is administered using a syringe pump (Graseby 3500; Graseby Medical Ltd., Walford, U.K.) via an intravenous fluid line. An IBM-compatible personal computer (PC) is used to calculate the infusion rate of propofol. These instruments are connected via RS-232 serial connections, and driven by software written by one of the authors (Y. Sawaguchi) in a commonly used computer language (Microsoft Visual C++ Ver. 6; Microsoft Corporation, Redmond, WA).

\section{B. Clinical Protocols}

After obtaining the approval of the Ethics Committee of the Kyoto University Graduate School and Faculty of Medicine, 160 adult patients [American Society of Anesthesiologists (ASA) physical status (PS) class I-II; age, 18-80 years; body mass index (BMI), $15-36 \mathrm{~kg} \mathrm{~m}^{-2}$ ] undergoing elective various kinds of ambulatory surgery were included in this study. They

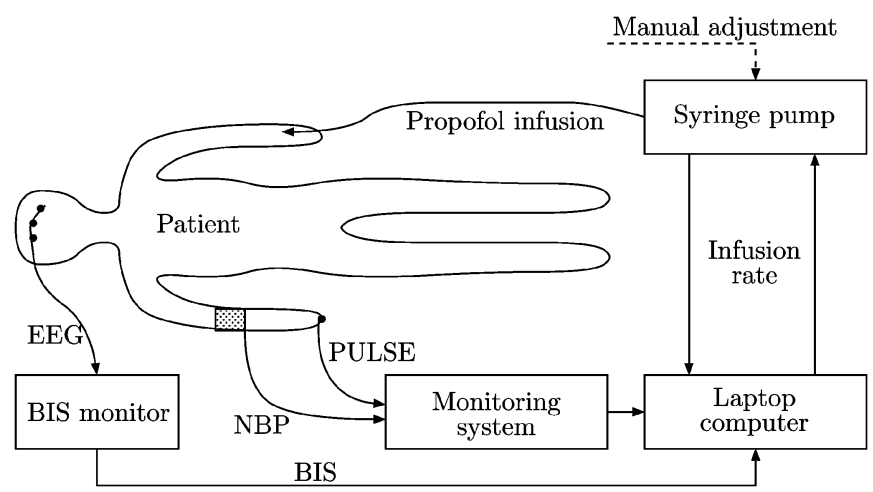

Fig. 6. Structure of our clinical trial system. The system comprises a BIS monitor, a patient monitoring system, a syringe pump, and a laptop computer. These devices are connected using RS-232 serial cables.

were assigned randomly to either group A (automatic control) or M (manual adjustment) using the envelope method.

Before induction, all patients were instructed to close their eyes. In group A, patients' hypnosis was controlled by our hypnosis control system, whereas patients in group $\mathrm{M}$ received propofol administration whose infusion rate was adjusted manually by one author (G. Shirakami) after $2-\mathrm{mg} / \mathrm{kg}$ initial bolus and $10-\mathrm{mg} / \mathrm{kg} / \mathrm{h}$ continuous infusion at an induction period. In both group, the target value of the BIS was set to 50 because the desired level of the BIS during surgery is 40-60 [15].

Supplemental IV fentanyl and vecuronium, local infiltration anesthetics, and rectal diclofenac were administered for pain relief and muscle relaxation during anesthesia. Boluses of fentanyl (25 $\mu \mathrm{g}$ each) were administered at the start of propofol infusion, before and $30 \mathrm{~min}$ after skin incision, and before skin closure. Vecuronium and rectal diclofenac were administered after loss of consciousness. The initial dose of vecuronium was $0.06-0.12 \mathrm{mg} / \mathrm{kg}$, depending on the estimated duration of anesthesia. Additional administration of vecuronium was done if insufficient muscle relaxation was observed. Local infiltration anesthetics were administered before the start and at the end of the operation by surgeons. In all cases, anesthesia care was provided by one author (G. Shirakami).

After each surgery, propofol infusion was terminated by the anesthesiologist. The recovery time from the stop of infusion to tracheal extubation or laryngeal mask airway (LMA) removal and the time required for the BIS to be above 80 after the stop of infusion were recorded.

In all cases, the BIS and infusion rate of propofol were recorded on a computer every second. Episodes of hypotension (systolic blood pressure $<70 \mathrm{mmHg}$ ) and bradycardia (pulse rate $<40 \mathrm{~b} / \mathrm{min}$ ) were also recorded. Evaluation and comparison of results were performed after all trials. Statistical analyses were performed using Student's $t$-test. Differences of $P<0.05$ were inferred to be statistically significant.

\section{Results}

In one case of automatic control, a misuse of the syringe pump engendered device failure. Consequently, that case was excluded from subsequent analyses.

Between the two groups, no statistically significant differences were found in patient characteristic data, type of surgery, 
TABLE III

PATIENT Characteristics, Performance Indices, Drug Consumption, AND OCCURRENCE OF ABNORMAL STATE OF AUTOMATIC CONTROL AND MANUAL ADJUSTMENT

\begin{tabular}{|c|c|c|}
\hline Group & A $(n=79)$ & $\mathrm{M}(n=80)$ \\
\hline Age (yr.) & $45.6 \pm 16.2$ & $47.4 \pm 16.4$ \\
\hline Height $(\mathrm{cm})$ & $160.1 \pm 8.2$ & $160.9 \pm 9.65$ \\
\hline Body weight $(\mathrm{kg})$ & $57.2 \pm 11.3$ & $56.0 \pm 11.2$ \\
\hline $\operatorname{Sex} \mathrm{m} / \mathrm{f}(n)$ & $19 / 60$ & $16 / 64$ \\
\hline ASA PS I/II $(n)$ & $52 / 27$ & $44 / 36$ \\
\hline Tracheal tube/LMA $(n)$ & $33 / 46$ & $28 / 52$ \\
\hline Duration of propofol infusion (min) & $107 \pm 45$ & $116 \pm 49$ \\
\hline \multicolumn{3}{|l|}{ Type of surgery } \\
\hline Breast surgery $(n)$ & 23 & 26 \\
\hline Oral surgery $(n)$ & 19 & 19 \\
\hline Hysteroscopic surgery $(n)$ & 17 & 16 \\
\hline Arthroscopic surgery $(n)$ & 8 & 6 \\
\hline Hernioplasty $(n)$ & 6 & 6 \\
\hline Dermatologic surgery $(n)$ & 4 & 3 \\
\hline Otolaryngologic surgery $(n)$ & 2 & 4 \\
\hline Averaged BIS & $48.4 \pm 2.5$ & $45.7 \pm 6.2^{* *}$ \\
\hline Averaged infusion rate $(\mathrm{mg} / \mathrm{kg} / \mathrm{h})$ & $8.28 \pm 2.0$ & $8.97 \pm 1.4^{*}$ \\
\hline Settling time (min) & $24.8 \pm 34.8$ & $37.1 \pm 49.8$ \\
\hline Time in target zone $(\%)$ & $78.1 \pm 15.3$ & $62.1 \pm 25.3^{* *}$ \\
\hline Time to BIS $=80$ after stop of infusion & $8.45 \pm 5.56$ & $7.46 \pm 3.86$ \\
\hline Time to extubation/LMA removal & $10.0 \pm 5.3$ & $8.75 \pm 3.98$ \\
\hline \multicolumn{3}{|l|}{$\begin{array}{l}\text { Drugs used during the maintenance } \\
\text { period }\end{array}$} \\
\hline Fentanyl $(\mu \mathrm{g} / \mathrm{kg})$ & $1.29 \pm 0.59$ & $1.18 \pm 0.82$ \\
\hline Vecuronium $(\mu \mathrm{g} / \mathrm{kg})$ & $125 \pm 44$ & $114 \pm 53$ \\
\hline Diclofenac $(n)$ & 62 & 64 \\
\hline Atropine $(n)$ & 1 & 0 \\
\hline Ephedrin $(n)$ & 0 & 1 \\
\hline \multicolumn{3}{|l|}{ Intraoperative episodes } \\
\hline Abnormal BIS rise ${ }^{\mathrm{a}}(n)$ & 71 & $51^{* *}$ \\
\hline Propofol bolus $(n)$ & 71 & $23^{* *}$ \\
\hline Hypotension $^{\mathrm{b}}(n)$ & 4 & 3 \\
\hline $\operatorname{Bradycardia}^{\mathrm{c}}(n)$ & 2 & 1 \\
\hline
\end{tabular}

Values are mean \pm SD or numbers $(n)$

a BIS $>65$

b Systolic blood pressure $<70 \mathrm{mmHg}$

c Pulse rate $<40 \mathrm{~b} / \mathrm{min}$

${ }^{*} p<0.05$

$p<0.01$

duration of propofol infusion, or consumption of drugs aside from propofol, as shown in Table III. In both groups, adequate anesthesia was achieved in all cases.

The BIS and the infusion rate in typical cases of automatic control and manual adjustment are shown, respectively, in Fig. 7(a) and (b). Fig. 7(a) shows the BIS and infusion rate for a 46-year-old male who underwent oral surgery. In this case, the parameter estimation was completed at $2.7 \mathrm{~min}$ after the start of infusion. The infusion was terminated at $138.8 \mathrm{~min}$. During the control period, defined as the period between the completion of the parameter estimation and the termination of infusion, the averages of the BIS and of the propofol infusion rate were 49.2 and $5.8 \mathrm{mg} / \mathrm{kg} / \mathrm{h}$, respectively. The settling time, defined as the time that the BIS is reached and is maintained within $40-60$ for
$5 \mathrm{~min}$, was $10.0 \mathrm{~min}$. The time in the target zone, defined by the percentage of time when the BIS is within 40-60 during the control period, was $90.8 \%$. The recovery time until extubation and the time required for the BIS to be above 80 after the stop of infusion were, respectively, 6.9 and $3.0 \mathrm{~min}$.

Fig. 7(b) also represents the BIS and infusion rate for a 57-year-old female patient who had oral surgery under manually adjusted propofol anesthesia which lasted $123.5 \mathrm{~min}$. For manually adjusted cases, we define the control period as the period from 4 min after the start of propofol infusion to the stop of infusion. The averages of the BIS and infusion rate during the control period were 51.7 and $9.8 \mathrm{mg} / \mathrm{kg} / \mathrm{h}$. The settling time, the time in target zone, and the recovery time to extubation and to $\mathrm{BIS}=80$ were, respectively, $22.8 \mathrm{~min}, 82.4 \%, 5.0 \mathrm{~min}$, and $12.3 \mathrm{~min}$.

The aggregated results of each group are shown in Fig. 8 and Table III. Fig. 8 presents the average and standard deviation of all time courses of the BIS and infusion rate before the stop of infusion. The averaged BIS is clearly closer to the setpoint, and the standard deviation of infusion rate is larger in our system. This means that our system can make the BIS closer to the setpoint taking individual differences of propofol requirement into account. In Table III, the mean values and standard deviations of the averaged BIS during the control period, the averaged infusion rate, the settling time, the time in target zone, and the recovery time to BIS $=80$ and tracheal extubation/LMA removal of each group are shown. Between the two groups, the averaged $\mathrm{BIS}$, the averaged infusion rate, and the time in target zone differ significantly. In group A, abnormal BIS rise and propofol bolus occurred more frequently than in group $\mathrm{M}$, whereas the frequency of hypotension and bradycardia did not differ between the two groups.

\section{DISCUSSION}

In this study, a model-predictive controller was developed for closed-loop control of hypnosis using propofol. The system is applied for ambulatory surgery because adverse reactions of propofol overdose are severe problems, especially for such situations. The proposed control framework combines the following: 1) a new description of propofol pharmacokinetics based on the result of Schüttler and Ihmsen [21], 2) a fast and reliable estimation of individual pharmacodynamic parameters, 3) a model-predictive controller that can easily incorporate information of a time delay and input constraints, and 4) a risk control function as a countermeasure to undesirable states. The model-predictive control strategy is a powerful method for controlling the pharmacological model of propofol; it achieves stable control in the presence of mismatches of time delay and gain. In the clinical trials, the model-predictive hypnosis control system can maintain patients' hypnosis adequately. Hereafter, we compare the results of clinical trials with those of previous works [5], [8]-[10], [12] and anesthesiologist's manual adjustment.

\section{A. Comparison With Other Closed-Loop Systems}

In most previous works, median performance error (MDPE) and median absolute performance error (MDAPE) were used to 

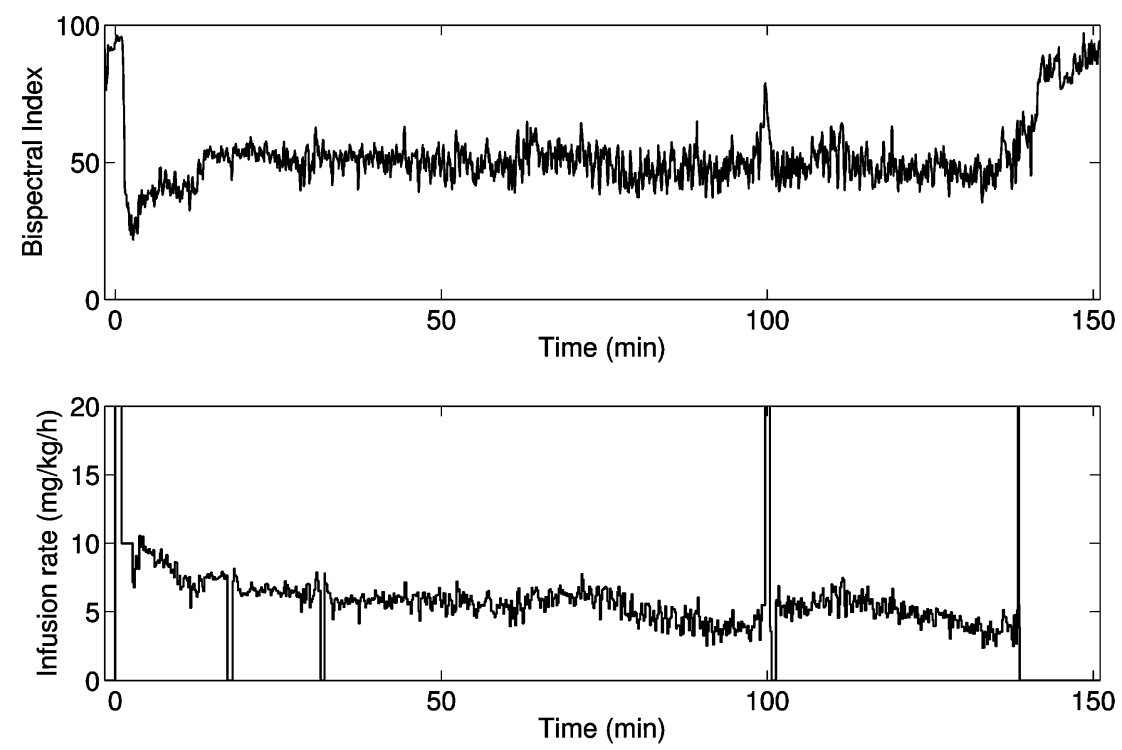

(a)
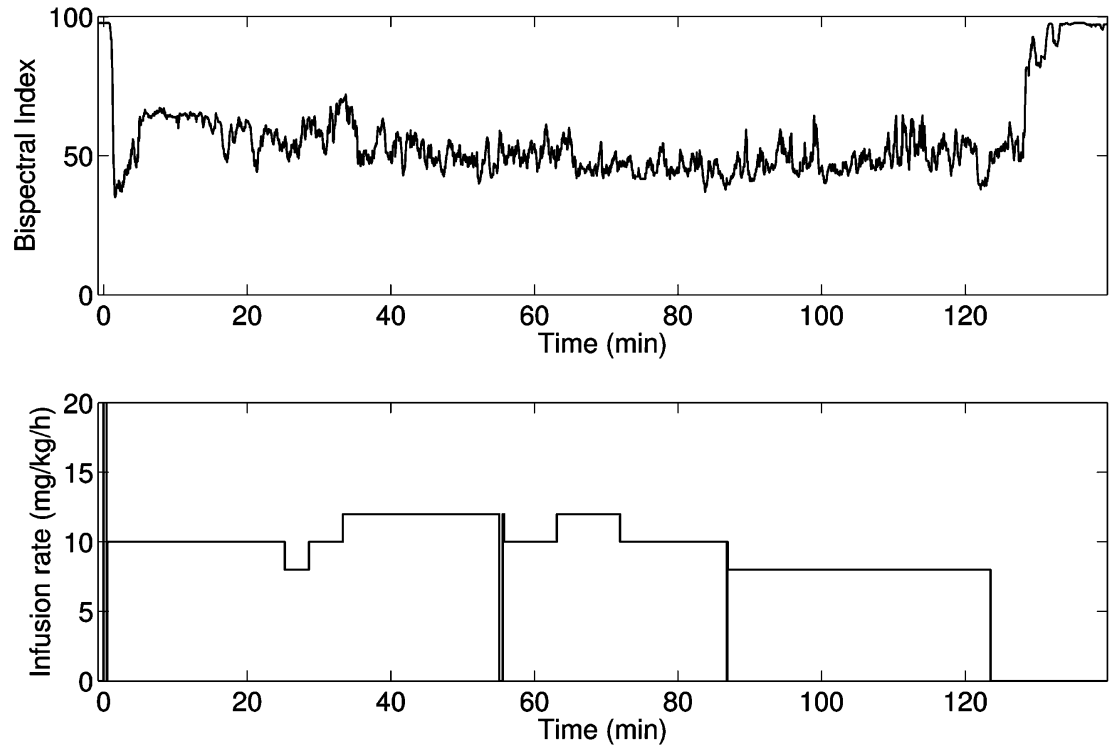

(b)

Fig. 7. Representative plots of both groups. In each figure, the upper and lower panels, respectively, show the BIS and infusion rate of propofol. (a) Typical result of automatic control (group A). (b) Typical result of manual adjustment (group M).

indicate the performance of the controller [5], [8]-[10], [12]. The MDPE and MDAPE are measures of performance error (PE), which are derived from

$$
\mathrm{PE}(t)=\frac{\mathrm{BIS}(t)-\text { BIS }^{\text {target }}}{\text { BIS }^{\text {target }}} \times 100 \% .
$$

The MDPE is a median of the PE, and the MDAPE is a median of the absolute values of the PE.

Table IV shows the MDPE and MDAPE of our system and those of previous works [5], [8]-[10], [12]. Table IV shows that the MDPE and the MDAPE of our system were not so good. A main reason for this is probably a pain relief method. In previous closed-loop systems, patients received epidural anesthesia
[8] or higher dose opioids [5], [9], [10], [12] than in our trials. For example, Absalom et al. [8] administered epidural anesthesia with $0.5 \%$ bupivacaine $10 \mathrm{~mL}$ and Liu et al. [10] and Struys et al. [12], respectively, used remifentanil infusion at the rates of about $0.22 \mu \mathrm{g} / \mathrm{kg} / \mathrm{min}$ and at least $0.25 \mu \mathrm{g} / \mathrm{kg} / \mathrm{min}$. In general, fluctuations of the BIS caused by painful stimulus depend on the level of analgesia [30]. Epidural anesthesia can produce dense sensory blockage; consequently, perturbation of the BIS during surgery can be suppressed. Intraoperative administration of opioid similarly affects the perturbation of the BIS [31]. However, epidural anesthesia requires a preparation time, causes delay of postoperative recovery, and poses risks of failure and accidental dural puncture. The high-dose administration of opioids causes adverse reactions such as PONV [32], which delays discharge from the hospital. Therefore, these analgesic 

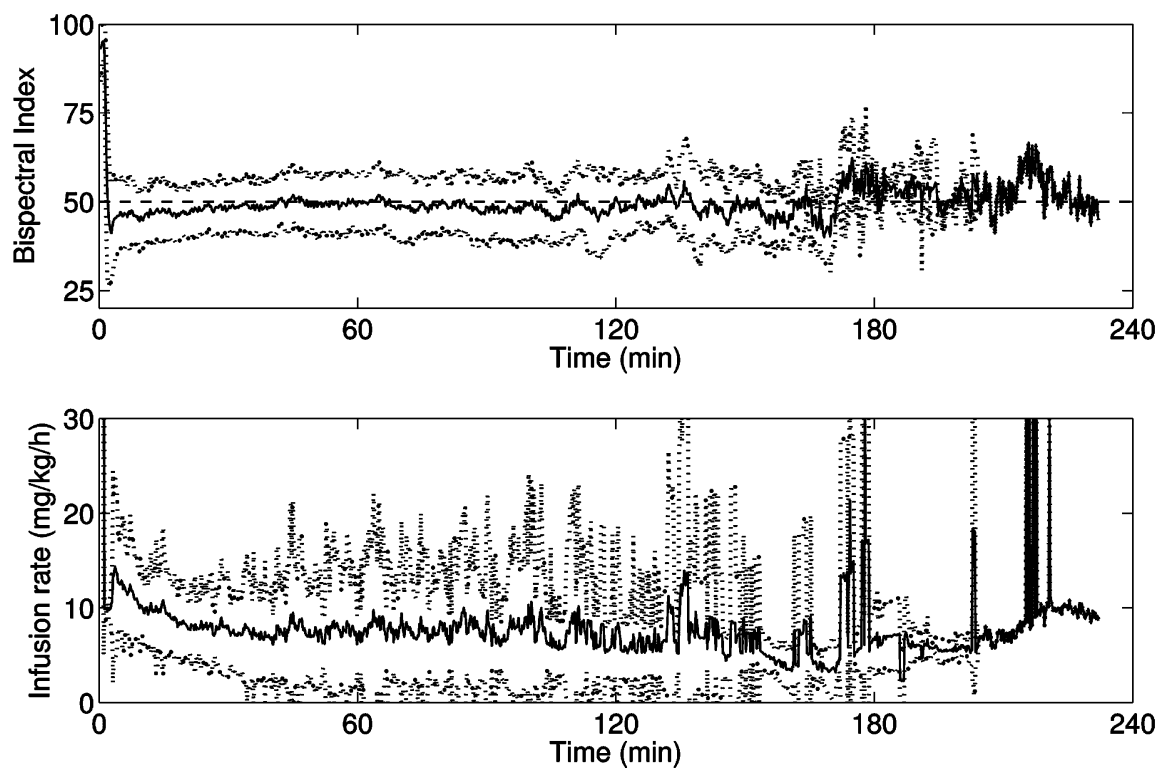

(a)
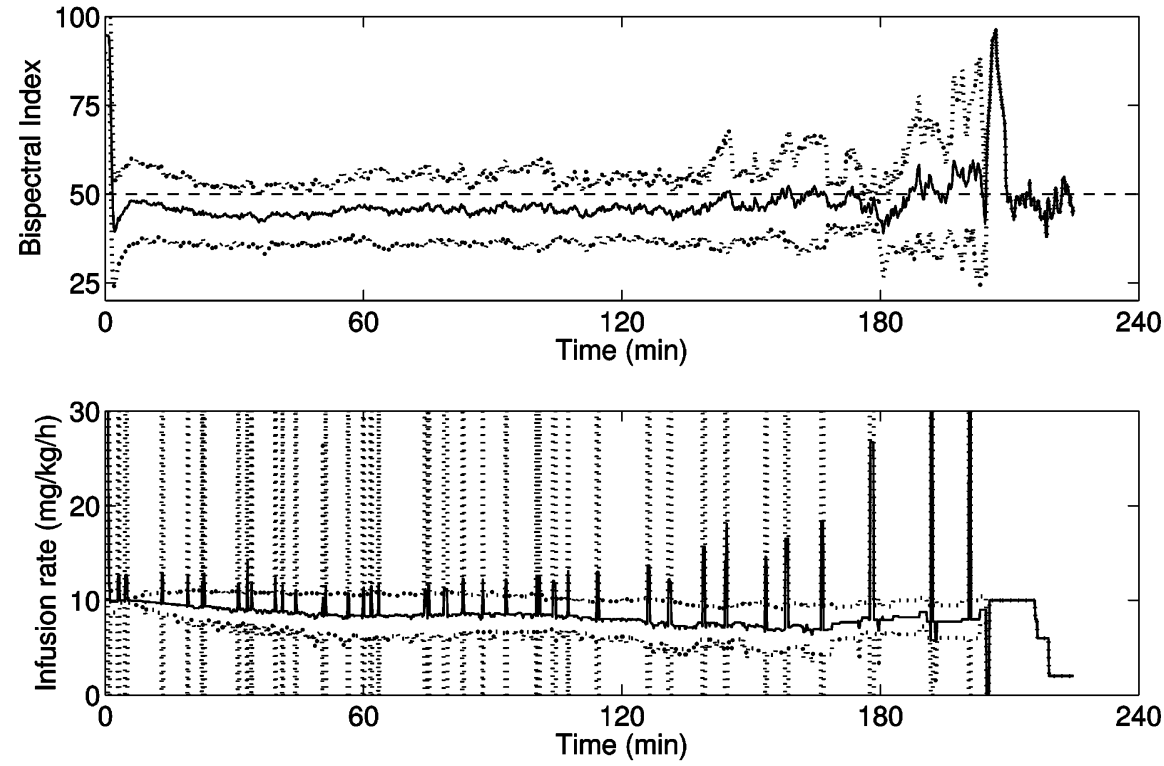

(b)

Fig. 8. BIS and infusion rate from induction to discontinuation of infusion for automatic control and manual adjustment groups. Average values (solid line) are presented with standard deviation values (dashed line). (a) Automatic control (group A). (b) Manual adjustment (group M).

methods are inappropriate for ambulatory surgery. On the other hand, we used intermittent fentanyl infusion for analgesia: its total amount was less than $100 \mu \mathrm{g}$. For that reason, our MDAPE was larger than others. For an appropriate comparison of the MDAPE, similar analgesic regimens must be used.

The MDPE of our system is not good, perhaps because of the use of the propofol bolus as a countermeasure to intraoperative arousal. The bolus was stopped when the BIS fell under 60 . However, the BIS continues to decrease after the stop of the bolus because of the time delay. Sometimes undershooting of the BIS to the target value of 50 occurs because the bolus dose is too large. To prevent an unnecessary BIS decrease, the effect of the delay must be considered adequately and the bolus dose
TABLE IV

MDPE AND MDAPE OF OUR SYSTEM AND OTHER PUBLISHED HYPNOSIS CONTROL SYSTEMS

\begin{tabular}{c||c|c|c|c}
\hline Developers & Analgesia & $n$ & MDPE & MDAPE \\
\hline \hline The authors & Fentanyl & 79 & -4.1 & 11.4 \\
\hline \hline Morley et al. [5] & Alfentanil & 30 & - & 13.6 \\
\hline Absalom et al. [8] & Epidural & 10 & 2.2 & 8.0 \\
\hline Absalom and Kenny [9] & Remifentanil & 20 & -0.42 & 5.63 \\
\hline Liu et al. [10] & Remifentanil & 83 & -3.3 & 9.9 \\
\hline Struys et al. [12] & Remifentanil & 10 & -6.6 & 7.7 \\
\hline
\end{tabular}

should be determined based on the future BIS predicted by a model with the consideration of disturbances. 
Another possible reason for the worse performance is the inaccuracy of the model. We have utilized a custom-made pharmacokinetic-pharmacodynamic (PK-PD) model. Although our PK-PD model has worked well, the model is not necessarily the best for prediction of the BIS response. This is because the model always has mismatches, which include individual differences in the PK-PD parameter values and estimation error in the pharmacodynamic parameters, with an actual patient. These mismatches yield bias and fluctuation of the BIS if the model is utilized in an open-loop controller. The closed-loop system can attenuate the effects of these mismatches. However, more accurate model improves the tracking ability and robust stability of the closed-loop control system. A possible candidate is a combined PK-PD model [26], whose plasma effect-site equilibration rate constant was derived in a more rigorous way.

\section{B. Comparison With Manual Adjustment}

As described in Section IV-C, the control system can maintain the BIS accurately and reduce the amount of propofol when compared to manual adjustments. These results are attributable to the accurate and adequate consideration of individual differences, the effect of time delays, and complex pharmacokinetics of propofol. However, some future directions of our control system are suggested by comparison with manual adjustments.

Before clinical trials, we anticipated that strict control of the BIS would decrease the propofol consumption and facilitate faster recovery from anesthesia. The averaged infusion rate is decreased as expected. However, the recovery profiles are almost identical to those of the manual adjustment cases, as shown in Table III.

A main reason for this lack of difference is the lack of consideration for the progress of the surgery. In the cases of manual adjustment, an anesthesiologist carefully monitors the patient state and the progress of the surgery. For example, the infusion rate would not be increased in the last stage of the surgery, even if a sudden rise of the BIS were to occur from the pain of skin sutures. On the other hand, our system only monitored the BIS, noninvasive systolic pressure, and pulse rate, and was unable to collect or use information about the progress of the surgery. In each clinical trial, the instructions to the controller given by the anesthesiologist were only two: "start infusion" and "stop infusion." Moreover, the target value, the control law, and the risk control rules during the control period were the same for all cases. Therefore, when a sudden BIS increase occurred because of skin sutures in the last stage of the surgery, the controller administered a bolus of propofol until the BIS fell to 60 . This BIS fall might have caused a delay of awakening. Changing the target point and risk control rules according to instructions about the progress of the surgery might bring about less drug consumption and earlier awakening.

\section{CONCLUSION}

In this study, a hypnosis control system was developed using a model-predictive controller. The system uses the BIS as the index of hypnosis and propofol as an anesthetic drug. It has a parameter estimation function of individual differences, in addition to a risk-control function. Results of clinical trials show the potential of our system in reducing the amount of propofol infusion and maintaining the BIS more accurately.

To improve the system effectiveness, we must consider the following topics:

- developing a more accurate model;

- improving a more reliable method for parameter estimation;

- optimizing rules for the risk control function.

\section{APPENDIX}

DERIVATION OF (12)

Subtracting each side of (8) from $E_{0}$ and multiplying them by $\left(y^{\gamma}(t)+c_{50}^{\gamma}\right)$, we obtain

$$
\delta_{\mathrm{BIS}}(t)\left(y^{\gamma}(t)+c_{50}^{\gamma}\right)=E_{\max } y^{\gamma}(t)
$$

where $\delta_{\mathrm{BIS}}(t)=E_{0}-\mathrm{BIS}(t)$. Transposing some terms, we get

$$
\left(E_{\max }-\delta_{\mathrm{BIS}}(t)\right) y^{\gamma}(t)=\delta_{\mathrm{BIS}}(t) c_{50}^{\gamma} .
$$

The logarithm of each side yields

$$
\begin{aligned}
\log \left[\left(E_{\max }-\delta_{\mathrm{BIS}}(t)\right) y^{\gamma}(t)\right] & =\log \left[\delta_{\mathrm{BIS}}(t) c_{50}^{\gamma}\right] \\
\log \left[E_{\max }-\delta_{\mathrm{BIS}}(t)\right]+\gamma \log [y(t)] & =\log \left[\delta_{\mathrm{BIS}}(t)\right]+\gamma \log \left[c_{50}\right]
\end{aligned}
$$

$\log \left[E_{\max }-\delta_{\mathrm{BIS}}(t)\right]-\log \left[\delta_{\mathrm{BIS}}(t)\right]=\gamma \log \left[c_{50}\right]-\gamma \log [y(t)]$

and (12).

\section{REFERENCES}

[1] E. Gepts, F. Camu, I. D. Cockshott, and E. J. Douglas, "Disposition of propofol administered as constant rate intravenous infusions in humans," Anesth. Analg., vol. 66, pp. 1256-1263, 1987.

[2] M. Tramer, A. Moore, and H. McQuay, "Propofol anaesthesia and postoperative nausea and vomiting: Quantitative systematic review of randomized controlled studies," British J. Anaesth., vol. 78, pp. 247-255, 1997.

[3] J. B. Glen, "The development of 'diprifusor': A TCI system for propofol," Anaesthesia, vol. 53, no. Suppl. 1, pp. 13-21, 1998.

[4] D. Russell, M. P. Wilkes, S. C. Hunter, J. G. Glen, P. Hutton, and G. N. Kenny, "Manual compared with target-controlled infusion of propofol," British J. Anaesth., vol. 75, pp. 562-566, 1995.

[5] A. Morley, J. Derrick, P. Mainland, B. B. Lee, and T. G. Short, "Closed loop control of anaesthesia: An assessment of the bispectral index as the target of control," Anaesthesia, vol. 55, pp. 953-959, 2000.

[6] K. Leslie, A. Absalom, and G. N. Kenny, "Closed loop control of sedation for colonoscopy using the bispectral index," Anaesthesia, vol. 57, pp. 693-697, 2002.

[7] T. Sakai, A. Matsuki, P. F. White, and A. H. Giesecke, "Use of an EEG-bispectral closed-loop delivery system for administering propofol," Acta Anaesthesiolog. Scand. vol. 44, pp. 1007-1010, 2000.

[8] A. R. Absalom, N. Sutcliffe, and G. N. Kenny, "Closed-loop control of anesthesia using bispectral index: Performance assessment in patients undergoing major orthopedic surgery under combined general and regional anesthesia," Anesthesiology, vol. 96, pp. 67-73, 2002.

[9] A. R. Absalom and G. N. Kenny, "Closed-loop control of propofol anaesthesia using bispectral index ${ }^{\mathrm{TM}}$ : Performance assessment in patients receiving computer-controlled propofol and manually controlled remifentanil infusions for minor surgery," British J. Anaesth., vol. 90, pp. 737-741, 2003.

[10] N. Liu, T. Chazot, A. Genty, A. Landais, K. McGee, P. Laloë, B. Trillat, L. Barvais, and M. Fischeler, "Titration of propofol for anesthetic induction and maintenance guided by the bispectral index: Closed-loop versus manual control: A prospective, randomized, multicenter study," Anesthesiology, vol. 104, pp. 686-695, 2006. 
[11] E. Mortier, M. Struys, T. D. Smet, L. Versichelen, and G. Rolly, "Closed-loop controlled administration of propofol using bispectral analysis," Anaesthesia, vol. 53, pp. 749-754, 1998.

[12] M. M. Struys, T. D. Smet, L. F. Versichelen, S. Van de Velde, R. Van de Broecke, and E. P. Mortier, "Comparison of closed-loop controlled administration of propofol using bispectral index as the controlled variable versus standard practice controlled administration," Anesthesiology, vol. 95, pp. 6-17, 2001.

[13] J. M. Bailey and W. M. Haddad, "Drug dosing control in clinical pharmacology," IEEE Control Syst. Mag., vol. 25, no. 2, pp. 35-51, Apr. 2005.

[14] J. C. Sigl and N. G. Chamoun, "An introduction to bispectral analysis for the electroencephalogram," J. Clin. Monit., vol. 10, pp. 392-404, 1994.

[15] T. J. Gan, P. S. Glass, A. Windsor, F. Payne, C. Rosow, P. Sebel, and P. Manberg, "Bispectral index monitoring allows faster emergence and improved recovery from propofol, alfentanil, and nitrous oxide anesthesia," Anesthesiology, vol. 87, pp. 808-815, 1997.

[16] M. Struys, L. Versichelen, G. Byttebier, E. Mortier, A. Moerman, and G. Rolly, "Clinical usefulness of bispectral index for titrating propofol target effect-site concentration," Anaesthesia, vol. 53, pp. 4-12, 1998.

[17] S. Pilge, R. Zanner, G. Schneider, J. Blum, M. Kreuzer, and E. F. Kochs, "Time delay of index calculation: Analysis of cerebral state, bispectral, and narcotrend indices," Anesthesiology, vol. 104, pp. 488-494, 2006.

[18] E. F. Camacho and C. Bordons, Model Predictive Control. London, U.K.: Springer-Verlag, 1999.

[19] Y. Sawaguchi, E. Furutani, G. Shirakami, M. Araki, and K. Fukuda, "A model predictive sedation control system under total intravenous anesthesia," in Proc. IEEE EMBS Asian-Pacific Conf. Biomed. Eng., 2003, pp. 358-359.

[20] E. Furutani, Y. Sawaguchi, G. Shirakami, M. Araki, and K. Fukuda, "A hypnosis control system using a model predictive controller with online identification of individual parameters," in Proc. IEEE Conf. Control Appl., 2005, pp. 154-159.

[21] J. Schüttler and H. Ihmsen, "Population pharmacokinetics of propofol-A multicenter study," Anesthesiology, vol. 92, pp. 727-738, 2000.

[22] S. L. Shafer and K. M. Gregg, "Algorithms to rapidly achieve and maintain stable drug concentrations at the site of drug effect with a computer-controlled infusion pump," J Pharmacokinet. Biopharm., vol. 20, pp. 147-169, 1992.

[23] H. Lange, H. Stephan, H. Rieke, M. Kellermann, H. Sonntag, and J. Bircher, "Hepatic and extrahepatic disposition of propofol in patients undergoing coronary bypass surgery," British J. Anaesth., vol. 64, pp. 563-570, 1990.

[24] C. F. Minto, T. W. Schnider, K. M. Gregg, T. K. Henthorn, and S. L. Shafer, "Using the time of maximum effect site concentration to combine pharmacokinetics and pharmacodynamics," Anesthesiology, vol. 99, pp. 324-333, 2003.

[25] J. G. Wagner, "Kinetics of pharmacologic response I. proposed relationships between response and drug concentration in the intact animal and man," J. Theor. Biol., vol. 20, pp. 173-201, 1968.

[26] T. W. Schnider, C. F. Minto, S. L. Shafer, P. L. Gambus, C. Andresen, D. B. Goodale, and E. J. Youngs, "The influence of age on propofol pharmacodynamics," Anesthesiology, vol. 90, pp. 1502-1516, 1999.

[27] J. Vuyk, F. H. Engbers, H. J. Lemmens, A. G. Burm, A. A. Vletter M. P. Gladines, and J. G. Bovill, "Pharmacodynamics of propofol in female patients," Anesthesiology, vol. 77, pp. 3-9, 1992.

[28] A. Shafer, V. A. Doze, S. L. Shafer, and P. F. White, "Pharmacokinetics and pharmacodynamics of propofol infusions during general anesthesia," Anesthesiology, vol. 69, pp. 348-356, 1988.

[29] S. Tonegawa, E. Furutani, and M. Araki, "Robust stability analysis of model predictive control systems for plants with a pure delay (in Japanese)," in Proc. 42nd Ann. Conf. Inst. Syst. Control Inf. Eng. (ISCIE), 1998, pp. 457-458.

[30] M. Jopling, R. C. Cork, and S. Greenwald, "Changes in the Bispectral Index (BIS) in the presence of surgical stimulation reflect the level of analgesia," Anesthesiology, vol. 84, pp. A478-A478, 1996.

[31] I. A. Iselin-Chaves, R. Flaishon, P. S. Sebel, S. Howell, T. J. Gan, J. Sigl, B. Ginsberg, and P. S. Glass, "The effect of the interaction of propofol and alfentanil on recall, loss of consciousness, and the Bispectral Index," Anesth. Analg., vol. 87, pp. 949-955, 1998.

[32] R. Sukhani, J. Vazquez, A. L. Pappas, K. Frey, and M. A. Slogoff, "Recovery after propofol with and without intraoperative fentanyl in patients undergoing ambulatory gynecologic laparoscopy," Anesth. Analg., vol. 83, pp. 975-981, 1996.

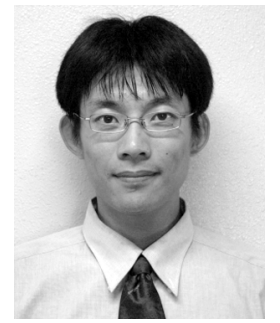

Yoshihito Sawaguchi (S'06-M'07) received the B.E. and M.E. degrees in electrical engineering from Kyoto University, Kyoto, Japan, in 2001 and 2003, respectively.

Currently, he is a Research Assistant at the Department of Control Engineering, Kisarazu National College of Technology, Chiba, Japan. His research interests are in the application of systems theory to biomedical topics such as signal processing of biomedical signals.

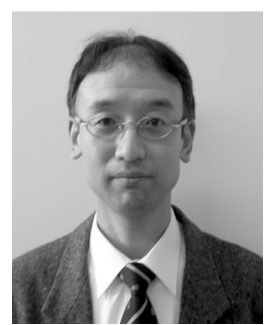

Eiko Furutani received the B.E., M.E., and Ph.D. degrees in electrical engineering from Kyoto University, Kyoto, Japan, in 1987, 1989, and 1997, respectively.

Currently, he is an Associate Professor at the Department of Electrical Engineering, Kyoto University. His research interests are in the application of control technologies to medical problems in corporation with medical doctors.

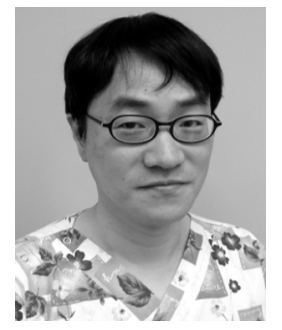

Gotaro Shirakami received the M.D. and Ph.D. degrees in medical science from Kyoto University, Kyoto, Japan, in 1984 and 1994, respectively.

Currently, he is an Associate Professor and Vice-Chair of the Department of Anesthesia, and a Vice-Director of the Division of Day Surgery, Kyoto University Hospital, Kyoto, Japan. His research interests are in ambulatory anesthesia and clinical significance of endogenous substances in cardiorespiratory regulation.

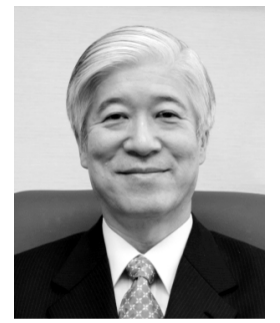

Mituhiko Araki (M'90-SM'03-F'06) received the B.E., M.E., and Ph.D. degrees in electronic engineering from Kyoto University, Kyoto, Japan, in 1966, 1968, and 1971, respectively.

From 1971 to 2006, he was with the Department of Electrical Engineering, Kyoto University. Since 2006, he has been serving as the President of the Matsue College of Technology, Institute of National Colleges of Technology, Shimane, Japan. His research interests are in systems and control theory and their applications including medical topics such as blood pressure control of patients under surgery.

Dr. Araki is the editor of Automatica for control system applications, Fellow of International Federation of Automatic Control (IFAC), and Professor Emeritus of Kyoto University.

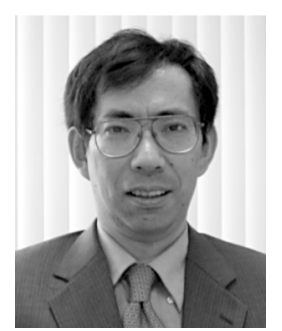

Kazuhiko Fukuda received the M.D. and Ph.D. degrees in medical science from Kyoto University, Kyoto, Japan, in 1981 and 1989, respectively.

Currently, he is the Professor and Chair of the Department of Anesthesia, Kyoto University, as well as the Director of the Division of Critical Care Medicine, Kyoto University Hospital, Kyoto, Japan. His research interests are in action mechanisms of opioid analgesics and inhaled anesthetics. 